\title{
Review of: "Deep Learning for Ultra-Widefield Imaging: A Scoping Review"
}

\author{
Gilbert Lim
}

Potential competing interests: The author(s) declared that no potential competing interests exist.

This is a comprehensive scoping review on the use of deep learning (DL) in ultra-widefield imaging (UWI). The authors have surveyed and categorized the available literature in the field, which currently comes to 36 studies. It appears an excellent resource for researchers interested in the field. A few comments might however be observed:

1. It is stated that machine learning (ML) networks "generate probabilities... by simulating the neural networks of human brains", but many ML models actually have no implicit inspiration from the brain. The taxonomy of ML vis-a-vis $\mathrm{DL}$ might be more precisely described.

2. "Steered images" are mentioned in passing, and might be briefly explained further.

3. For the methodology, the inclusion criteria might be more precisely specified (i.e. search in title, abstract, etc.) if possible.

4. It is stated that data augmentation can increase the size of the training set manyfold. It might be noted that on-the-fly image augmentation can technically produce a different image instance, each time a particular image is accessed for training.

5. Some studies appear to possibly fall under multiple categories (e.g. GAN synthesis with disease classification). This might be considered to be reflected.

6. The data in Tables $1 \& 2$ appear to be truncated currently. It might be considered to present these tables in landscape orientation, and possibly in a smaller font.

7. The working resolution of the images for each study might be a useful inclusion in the relevant table(s).

8. A couple of minor corrections/rephrasings might be considered, e.g. "A literature search was performed up to August 31st, 20201", "The AUROC value serves as a measure of the model to correctly classify and predict based on the test data". 